\title{
Z Research Square \\ Profiling Gut Microbiota and Bile Acid Metabolism in Critically ill Children
}

\author{
University of Cambridge \\ Joseph Wagner \\ Melbourne Health \\ Anisha Wijeyesekera \\ University of Reading \\ Marcus De Goffau \\ University of Cambridge

\section{Sarah Thurston} \\ University of Cambridge \\ John A. Clark \\ University of Cambridge \\ Deborah K. White \\ University of Cambridge \\ Jenna Ridout \\ $\mathrm{EACH}$
}

lain Robert Louis Kean ( $\nabla$ irlk2@cam.ac.uk)

\section{Shruti Agrawal}

Cambridge University Hospitals NHS Foundation Trust, Addenbrooke's Hospital

Riaz Kayani

Cambridge University Hospitals NHS Foundation Trust, Addenbrooke's Hospital

\section{Roddy O'Donnell}

Cambridge University Hospitals NHS Foundation Trust, Addenbrooke's Hospital

\section{Padmanabhan Ramnarayan}

Great Ormond Street Hospital for Children NHS Foundation Trust

\section{Mark J. Peters}

UCL Great Ormond Street Institute of Child Health

Nigel Klein

UCL Great Ormond Street Institute of Child Health

\section{Elaine Holmes}

Imperial College London

Julian Parkhill

University of Cambridge

\section{Stephen Baker}


University of Cambridge

\section{Nazima Pathan}

University of Cambridge

\section{Research Article}

Keywords: paediatric, microbiome, critical illness, bile acid

Posted Date: December 3rd, 2021

DOI: https://doi.org/10.21203/rs.3.rs-1111591/v1

License: (c) (1) This work is licensed under a Creative Commons Attribution 4.0 International License. Read Full License 


\section{Abstract}

Broad-spectrum antimicrobial use during the treatment of critical illness influences gastrointestinal fermentation endpoints, host immune response and metabolic activity including the conversion of primary to secondary bile acids. We previously observed reduced fermentation capacity in the faecal microbiota of critically ill children upon hospital admission. Here, we further explore the timecourse of the relationship between the microbiome and bile acid profile in faecal samples collected from critically ill children.

The microbiome was assayed by sequencing of the 16s rRNA gene, and faecal water bile acids were measured by liquid chromatography mass spectrometry. In comparison to admission faecal samples, members of the Lachnospiraceae recovered during the late-acute phase (days 8-10) of hospitalisation. Patients with infections had a lower proportion of Lachnospiraceae in their gut microbiota than controls and patients with primary admitting diagnoses. Keystone species linked to ecological recovery were observed to decline with the length of PICU admission. These species were further suppressed in patients with systemic infection, respiratory failure, and undergoing surgery. Bile acid composition recovers quickly after intervention for critical illness which may be aided by the compositional shift in Lachnospiraceae. Our findings suggest gut microbiota recovery can be readily assessed via measurement of faecal bile acids.

\section{Introduction}

The establishment of a healthy gut microbiota in early life is considered to be protective against a range of allergies and communicable and non-communicable diseases ${ }^{1-8}$. Multiple factors, such as delivery method, feeding, diet, illness, age, and antimicrobial use in early life, can impact on the establishment and maintenance a healthy gut microbiota $1,2,8,9$. The administration of broad-spectrum antimicrobials is often necessary during hospitalisation for critical illness, which reduces the diversity of the gut microbiota ${ }^{3}$. The effects of broad-spectrum antimicrobial therapy on the microbiota, and the subsequent recovery process, have been well documented ${ }^{10-12}$. However, critical illness can have profound effects on feeding and energy intake ${ }^{13}$, and respiratory complications may lead to low oxygen saturation and subsequent hypoxia, inducing disruption of the gut microbiota ${ }^{14}$. Furthermore, changes in the structure of the microbiota may exacerbate the disease state or alter the course of recovery $3,12,15$. The body of evidence identifying illness as both a cause and effect of an altered microbiota is becoming more extensive ${ }^{9}$.

Bile acids shape the composition of the microbiota and correspondingly the structure of the microbiota contributes to the metabolism of the bile acids ${ }^{16}$. Notably, the conversion from primary to secondary bile acids requires a cascade of enzymatic modifications incorporating a 7a-dehydratase enzyme expressed by a range of Gram-positive bacteria ${ }^{17,18}$. Further metabolism of bile acids by intestinal bacteria enhances passive reabsorption by the intestine; therefore, the microbiota also contribute to the recycling 
of bile acids ${ }^{19}$. Secondary bile acids can also inhibit intestinal pathogens and have been shown to restrict the germination and sporulation of Clostridioides ${ }^{20,21}$. The relationship between the microbiome and disease is complex; altered bile acid profiles are associated with diseases such as metabolic syndrome and colon cancer, which may also be exacerbated by the microbiota ${ }^{22-26}$. The western diet has been associated with higher levels of taurocholic acid, hydrogen sulphide and deoxycholic acid, which have been linked to ulcerative colitis and colon cancer ${ }^{24,27-30}$. Bile acids modulate the farnesoid $X$ receptor and G-protein-coupled bile acid receptor, with both receptors playing active roles in glucose and lipid homeostasis as well as insulin sensitivity 25,26 .

We previously characterised the functional capacity of the intestinal microbiome by exploiting multicompartmental metabolic profiling ${ }^{10}$, and observed a shift in the short chain fatty acid and faecal bile acids profiles in critically ill children compared to healthy age-matched controls. Our previous work examined the acute changes in faecal microbiome in children admitted to the Paediatric Intensive Care Unit (PICU) for critical illness. Here we examine differences in the microbiome of critically ill children on ventilators during different stages of hospitalization for critical illness and the associated impact on total faecal bile acid composition.

\section{Results}

\section{Demographics}

We examined samples from 67 mechanically ventilated critically ill children and 52 age-matched healthy controls. The critically ill children were aged between 1 and 16.9 years with a median age of 4.1 years (inter-quartile range (IQR); 8.5 years). The healthy controls were aged between 1 and 15.9 years, with a median age of 5.8 years (IQR; 2.6 years) $(p=0.31)$. The median weight of the critically ill children was 15.7 $\mathrm{kg}$, (IQR; $13.9 \mathrm{~kg})$; the healthy controls had a median weight of $20.4 \mathrm{~kg}(\mathrm{IQR} ; 9 \mathrm{~kg})(p=0.005)$. The median number of PICU free days within 30 days of admission in the critically ill children was 22 (IQR; 8 days) and the median number of days free of invasive mechanical ventilation within 30 days of admission was 26 days (IQR; 4.5 days). The survival rate was $96 \%$. Further demographic is shown in Tables S1 and Table S2 (Kean_2021_supplementary_tables.pdf).

Bile acids

We analysed the composition of faecal bile acids and the relative concentrations of different bile acid groups using LC-MS on 43 stool samples collected from 39 critically ill children and 40 samples from healthy controls. The critically ill children sampled on day 1-3 and day 4-7 had significantly higher concentrations of primary bile acids in their faeces compared than the control group $(p<0.001, p<0.001)$ (Figure 1a). Additionally, the concentration of primary bile acids were significantly lower at day 8-10 than earlier in the course of admission (day 1-3 samples) $(p=0.008)$. The inverse trend was observed for the secondary bile acids (Figure 1b). 
We examined the ratio of different bile acids. We observed a significant change in the ratio of the two primary bile acids, (cholic acid and chenodeoxycholic acid; $C A: C D C A$ ) in all patient sampling timepoints relative to the control (Figure 1c). The primary/secondary bile acid ratios in critically ill patients were significantly higher at day 1-3 and day 4-7 compared to controls $(p<0.001, p<0.001)$. Conversely, there was a significant reduction in the primary/secondary bile acid ratios in the samples taken on days 8-10 compared to the control (Figure 1d). The ratios of cholic acid to deoxycholic acid (CA:DCA) measured in patient sample faecal water significantly increased on day 1-3, day 4-7 and days $8-10$, compared to the control samples but the median ratio on days 8-10 was significantly lower than the median of day 1-3 and day 4-7 (figure 1e). A significant change was also observed in in the ratio of chenodeoxycholic acid to total lithocholic acid (CDCA:LCA) between the control group and day 1-3 and day 4-7 (figure 1f).

To assess bile acid differences at different sampling periods, we compared the relative concentration of individual bile acids (Figure S1 Kean_2021_Figure_S1.eps). Secondary bile acids were frequently more abundant in control samples than at day 1-3 and day 4-7. However, the median relative concentration of each bile acid from patients sampled at day 8-10 shifted towards the median of the control group for the majority of bile acid interrogations (Figure 1), Non-DCA/LCA bile acids were more abundant in healthy controls; Hydroxy- and Oxo- modifications at carbon 3 and 12 are reversible moieties catalysed by the gut microbiota, in addition to the well-established 7a-dehydratase conversion of CA to DCA and CDCA to LCA/ILCA.

\section{Bacterial populations}

Children admitted to a PICU are frequently administered broad spectrum antimicrobials soon after admission. In this study, 42/43 (97.7\%) PICU patients whose faecal microbiome was assessed by $16 \mathrm{~s}$ rRNA gene sequencing, received antimicrobial therapy during the course of PICU admission. Applying a Kruskal-Wallis chi-squared test, no significant difference was observed in the number of antimicrobials given to patients across all sampling groups ${ }^{10}$.

To investigate microbial variation between individuals and groups, alpha and beta diversities were examined. Quantifying alpha diversity using Shannon's Index, we observed greater median diversity with a narrow range in the control group, compared to all patient sampling time points (Figure 2a). To determine if groups were compositionally different, beta diversity was calculated using Bray-Curtis dissimilarity. Using NMDS plotting we observed that all patient sample groups significantly diverged from the control group based on Bray-Curtis dissimilarity (figure $2 b$ ). The difference between groups was significant by PERMANOVA, performed using adonis from the vegan package in $R(p=0.0001)$. To identify intergroup significance, we applied Tukey's Honest Significant Differences (HSD) post hoc test to a principal coordinate analysis of the Bray-Curtis dissimilarity matrix, resulting in adjusted p-values of $<0.0001$ for all pairwise comparisons between the control and patient groups (data not shown). Intergroup analysis using Tukey's HSD identified no difference between patient samples ( $p>0.95$ for all groups). The bacterial population profiles of study participants, represented at the Family level, are shown in Figure 3. 
Bacteria associated with gut microbiome recovery have previously been characterised in studies observing the fluctuation in microbiota induced by antimicrobial use ${ }^{12}$. Bacterial genera Alistipes, Bacteroides, Bifidobacterium, Coprococcus, Desulfovibrio, Faecalibacterium, Parabacteroides, Roseburia, Ruminococcus and Subdoligranulum have been previously described as recovery associated bacteria (RAB). Observing these genera as a group, the general proportion of all RAB significantly decrease from control samples across all patient sampling timepoints (Figure 4a). The loss of RAB was related to the time in which the sample was collected (Figure S2a Kean_2021_Figure_S2.eps). No correlation was observed between RAB proportion and number of antimicrobials administered prior to sample collection $(\rho[51]=-0.058, p=0.6854)$. Weak negative correlations were observed between RAB proportion and hospital days before sample collection, but this trend was not significant $(\rho[51]=-0.217, p=0.123)$. RAB were decreased relative to controls for patients admitted with non-respiratory infections, patients with noninfectious respiratory failure, and surgical patients (Figure S2b).

We observed a decrease in the proportions of RAB as PICU stay lengthened, with most samples collected at days 8-10 containing $<50 \%$ RAB by relative proportion (Figure $4 a$ ). RAB proportion reduced with sample produced, where first samples had higher abundance or RAB than the second or third stool sample collected. (Figure S2a). RAB were also higher for all admitting diagnosis groups except for Neurological admissions (Figure S2b). The Individual RAB comparisons are shown in Figure S3 (Kean_2021_Figure_S3.eps). Bacteroides spp. contributed the largest proportion of gut bacteria in the control group (Figure S3b). The patient group displayed a wide range of Bacteroides abundance across sampling ranges; however, the median abundance was significantly below that of the control group for all time frames (Figure S3b). The majority of patient samples were comprised of $<25 \%$ Bacteroides. Of the RAB, Faecalibacterium spp. and Ruminococcus spp. both contributed similarly to the proportion of the control group (Figure S3). A significant reduction was observed between the control faecal microbiomes and patient faecal microbiomes for both genera $(p=0.001, p=0.004, p<0.001)$. The amount of Faecalibacterium during all timepoints in PICU stays was dramatic, with an average proportion of $0.25 \%$ in patient samples versus $6.7 \%$ in control samples. This observation is suggestive of extensive antimicrobial susceptibility among the Faecalibacterium. A significant reduction in abundance of Ruminococcus spp. was observed in all patient groups compared to control microbiome abundances.

The metabolism of primary bile acids to secondary bile acids is classically performed by members of the Clostridium and Eubacterium. To determine if there was a link between secondary bile acid recovery and the microbiome, we measured the correlation between secondary bile acids and bacterial proportions for patient samples that were subjected to both analyses $(n=15)$. Total secondary bile acids correlated weakly $(0.20 \leq \rho<0.4)$, with the observed proportion of Clostridium sensus stricto group 1 ( $\rho[15]=0.205$, $p=0.446)$. The genus Clostridioides was moderately correlated $(0.40 \leq \rho<0.6)$ with $D C A(\rho[15]=0.483, p=$ $0.068)$. Assessing the correlation between the abundances of bacterial genera and major secondary bile acid relative concentrations (DCA and total LCA/ILCA) (Table S3), we observed a strong correlation between DCA and Agathobacter, Anaerovorax and the Eubacterium oxidoreducens group across all patient samples with paired bile acid profiles and quantified microbiomes (Table S3). The genera Anaerovorax, Gordonibacter, Romboutsia, Solobacterium and, Turicibacter were strongly correlated with 
total LCA and DCA. Four bacterial genera with moderate positive correlation (Table S3) were from the family Lachnospiraceae.

The genera within the Lachnospiraceae identified in this study were Agathobacter, Anaerobutyricum [Eubacterium] hallii group ${ }^{31}$, Anaerocolumna, Anaerostignum, Anaerostipes, Blautia, Butyrivibrio, Coprococcus, Dorea, Eisenbergiella, Fusicatenibacter, Howardella, Hungatella, Lachnoanaerobaculum, Lachnoclostridium, Lachnospira, Mediterraneibacter[Ruminococcus] gnavus group ${ }^{32}$, Mediterraneibacter [Ruminococcus] torques group, Oribacterium, Pseudobutyrivibrio, Roseburia, Stomatobaculum and Tyzzerella. There was no significant difference in the proportional representation of the Lachnospiraceae between patient groups (Figure 4b). A shift in the composition of the Lachnospiraceae was observed when the constituent genera were examined (Figure S4); Heterogeneous, Agathobacter dominant control profiles were replaced with Lachnoclostridium and A. hallii group bacteria in patient profiles (Figure S4 Kean_2021_Figure_S4.eps).

We examined the proportion of individual Lachnospiraceae genera for correlation with other bacteria groups in samples from day 8-10. Where a correlation was identified, Ruminococcus and Solobacterium were most often positively correlated to genera of Lachnospiraceae. There was a negative correlation between Enterococcus and Anaerobutyricum hallii group, Dorea, Lachnoclostridium, Mediterraneibacter gnavus group, and Tyzzerella (Table S4).

Comparing the median proportions of Lachnospiraceae across all major we observed a decreased median proportion for respiratory infections, and non-respiratory infections. The difference was not significant after correction for multiple comparisons across six groups (Figure S2c). Patients with respiratory infections had significantly lower proportions of Lachnospiraceae than healthy controls when compared to all other diagnoses (Figure S2d). When patients were admitted for a non-respiratory infection, the Lachnospiraceae were also significantly decreased from the control group (Figure S2e).

\section{Discussion}

In this study, we identify divergent faecal bile acid profiles between patients and healthy controls; these differences may prove useful clinical markers of gut health and recovery. In our study group, day 1-3 and day 4-7 patient sampling time ranges had median primary bile acid compositions significantly above the control group while patients sampled during days 8-10 of PICU stay had bile acid compositions comparable to healthy control. The greater proportion of CA, and CDCA may be attributed to the reduction of intestinal bacteria capable of bile acid conversion 23,33-35; concordantly, the inverse was observed for secondary bile acids. It is possible that the apparent recovery of bile acid metabolism by the end of PICU stays may reflects early-stage microbiome recovery. A future study with later timepoints would be needed to confirm this observation.

The composition of the gut microbiota was significantly affected during critical illness. Differences in microbial composition and richness between controls and critically ill patients were observed in this 
study. Decreasing alpha-diversities at varying body sites during hospitalisation in paediatric intensive care facilities has been previously reported by Rogers et al. ${ }^{11}$. An expected decrease in microbial richness, quantified by Shannon's index, can be observed as the length of PICU stay increases. Treatment with broad-spectrum antimicrobials will be the driver for the compositional change and loss of diversity. Beta diversity varies widely in all treatment groups compared to control samples, and as expected there is a difference in faecal microbiome beta diversity between the control and sampling groups, most likely driven by antimicrobial treatment. A study by Chng and colleagues identified bacteria linked to microbiome recovery after antimicrobial therapy ${ }^{12}$. These RAB may act as keystone species ${ }^{36,37}$, providing metabolic substrates for endpoint fermenters such as the Lachnospiraceae. The general trend for RAB was to decrease in proportion with treatment length. The loss of these species in over time in our study is most likely explained by the duration of action of antimicrobial therapy. Decreased motility may account for some protection of RAB bacteria from antimicrobial killing, or the withdrawal of antibiotics but continued hospitalization may have allowed for recovery. Reduced gastric motility during critical illness can hinder sample collection. Patients in this study may not have had a first sample collected until 10 days post-admission.

Treatment for critical illness drives a compositional change in the Lachnospiraceae, with a slight decrease in median abundance. The observed increase in secondary bile acids in patients sampled at day 8-10 could be driven by a change in Lachnospiraceae, specifically, bacteria related to Lachnoclostridium and the $A$. hallii group bacteria ${ }^{38-40}$. Bacteria from Lachnoclostridium and the $A$. hallii group were present ( $>10$ counts) in 7 of 11 patient samples at days 8-10. Other bacteria in the family Lachnospiraceae were minor contributors in the microbiota of patients at days 8-10, with the exception of patient 18. Compositional changes of the Lachnospiraceae may be driven by changes in the upstream fermentation capacity of patient microbiota leading to increased production of mid-point fermentation products by the new dominant microbiota of critically ill children ${ }^{10}$. Bacteria in the A. hallii group and Lachnoclostridium are capable of utilising lactate produced by Escherichia coli, Bifidobacterium, Lactobacillus, and Enterococcus ${ }^{41}$. Further interpretation of the connection between bile acid production at days 8-10 and the microbiota is limited due to the small overlap in patients with both microbiomes and bile acid profiles $(n=2)$. An average PICU stay in the United Kingdom lasts 2-3 days, across all ages 42 . Samples collected at later timepoints are often from the sickest patients. The study is limited by the inability to follow the microbiome and bile acids of patients post-discharge.

Members of the Lachnospiraceae are known to metabolise primary to secondary bile acids through a 7adehydratase process ${ }^{39}$. The selection of enrichment for $A$. hallii group bacteria may be due to their ability to use both D- and L-lactate ${ }^{41}$. Increased total lactate was indicated as a measure of critical illness ${ }^{10}$. Members of the Clostridiaceae and Ruminococcaceae were not as abundant as the Lachnospiraceae at days 8-10, therefore unlikely to drive bile acid conversion.

An alternative hypothesis is that members of the Lachnospiraceae are differentially resistant to antibiotics, and strains are expanding to fill empty ecological niches. The interpretation of differential 
resistance is limited due to the use of 16s rRNA gene profiling and lack of information on the distribution of antimicrobial resistance genes. The shift in Lachnospiraceae composition from control to patient profiles appears as early as the first day of PICU admission. Duration of antimicrobial therapy and classes of antimicrobial can be difficult to quantify in a PICU setting; patients may have received antimicrobial therapy prior to admission, where information about treatment may held by another hospital and not readily available. After antimicrobial therapy certain bacteria recover more rapidly than others, contributing to the total biomass of faeces. We observed no strong effect due to change in faecal biomass, or biomass recovery when using contamination of 16s rRNA gene sequences derived from DNA extraction kits as a proxy for bacterial biomass.

During early life, the composition of the microbiome changes substantially, from species acquired during delivery, through breastfeeding and the transition to different foods. Studies have linked the method of delivery to an altered microbiome, children born by Caesarean section are at greater risk of autoimmune disease compared to children delivered via normal vaginal delivery ${ }^{1,43-45}$. As children mature, microbial complexity increases and "healthy" microbiomes are associated with greater bacterial diversity, with Bacteroidetes and Firmicutes making up the majority of the proportional representation ${ }^{46}$. The general trend in our study was that healthy children had richer microbial diversity and high proportions of Firmicutes and Bacteroidetes. Children requiring hospitalisation in PICUs often had highly disrupted microbial communities when compared to healthy children. Upon arrival to PICU, patients have most often received a course of antimicrobial therapy or will be treated with a broad-spectrum antimicrobial such as ceftriaxone. The effect of these treatments confounds the ability to interpret the impact of critical illness on the microbiome in the absence of internal reference.

Previously, we examined paediatric critical illness from a multi-component perspective and demonstrated that critical illness affects the microbiome of paediatric patients, which is consistent with other similar studies ${ }^{10}$. Faecal metabolic profiling indicated a higher correlation of sugars and lactate in patients, with short chain fatty acids increased in age matched controls. A brief examination of bile acids by Wijeyesekera et al. ${ }^{10}$ directly linked levels of the secondary bile acids DCA, LCA and ILCA to the proportion of Bacteroides, Ruminococcus, Eubacterium, Lachnospiraceae, and Faecalibacterium in faeces. Our results indicate a positive correlation in patient samples between secondary bile acids and the genera Anaerovorax, Barnesiella, Faecalitalea, Gordonibacter, Megamonas, Odoribacter, Parasutterella, Pseudoflavonifractor, Solobacterium and Turicibacter, however, the limited number of patient samples with both a quantified microbiome and a bile acid profile limits the predictive power of these observations.

Currently, profiling of microbiomes by $16 \mathrm{~s}$ rRNA gene sequencing or shotgun metagenomics is a costand labour-intensive process. Even using only nanopore sequencing devices involves multiple steps. Days of data collection and processing are required to obtain a snapshot of the microbiome. The measurement of metabolomes by mass spectroscopy is rapid (under 30 minutes) and can be performed with minimal preparation from biological samples ${ }^{10,47,48}$. Clinical diagnostic laboratories may have 
services using mass spectroscopy as standard analytical techniques ${ }^{49,50}$. Measurement of primary and secondary BAs from blood, urine and faeces may therefore be within the capabilities of modestly outfitted laboratories.

The development of a healthy microbiome early in life is considered essential to systemic health $3,4,51,52$. If the microbiome fails to recover quickly, or enters an altered state, there may be differential bile acid metabolism, SCFA production, and overall altered microbial metabolic interactions. The recovery of the microbiota is dependent on the diversification of metabolic interactions after antibiotics ${ }^{12,53}$. Current predictive modelling programs attempt to predict host metabolomes from gut microbiomes ${ }^{54,55}$ and it may be possible to estimate the recovery of the microbiome based on metabolomics alone.

The power of this study to predict bacteria contributing to the recovery of bile acid profiles is limited by the overlap of patients with both microbiomes and bile acid profiles.

\section{Conclusion}

Our study observed relative concentrations of secondary faecal bile acids to be comparable with those of healthy controls after hospitalization in a PICU for 8-10 days. Compositional proportions of Bacteroides, Ruminococcus, and Fecalibacterium were reduced in microbiomes at day 8-10, but proportional representation of Lachnospiraceae remained close to control levels across all time ranges. Monitoring of faecal metabolites (SCFAs, sugars) and bile acids provides a rapid and cost-effective approach to determine intestinal microbiome health and recovery. Further studies are warranted to link markers of microbiome recovery after intervention for critical illness with clinical significance.

\section{Materials And Methods}

In this study we further examined the data collected by Wijeyesekera et al. ${ }^{10}$.

\section{Study participants}

In this prospective, multi-centre cohort study, critically ill children were recruited if they were admitted to one of three paediatric intensive care units (PICUs) in the North Thames Region (UK). Following informed consent by caregivers, children aged between 1 and 16 years were eligible if they required mechanical ventilation. Children with chronic illness or prolonged steroid use were excluded. Healthcare data was acquired from hospital medical records. In order to compare data with healthy controls, faecal samples were obtained from age-matched children, who were recruited from the local community. These children were eligible if they were free of intercurrent illness, had a normal healthy diet, and had not received antibiotics in the prior 3 months.

Approval for the study was granted by the East Midlands-Nottingham 2 Research Ethics Committee for recruitment from the PICUs at Cambridge University Hospitals NHS Foundation Trust, Great Ormond 
Street Hospital NHS Foundation Trust, and Imperial College Healthcare NHS Foundation Trust. The City Road and Hampstead Research Ethics committee approved recruitment of healthy children in the Cambridge vicinity. REC reference: 13/LO/0974. All methods were performed in accordance with the relevant guidelines and regulations.

\section{Faecal samples}

Faecal samples were collected at early (within first 3 days of PICU admission), mid (days 4-7 of PICU admission), and late timepoints (days 8-10 of PICU admission) from critically ill children. A single faecal sample was collected from healthy children. All samples were taken from nappies, placed in sterile plastic containers, and stored at $-70^{\circ} \mathrm{C}$ until use.

\section{Taxonomic data}

We performed sequencing of the bacterial 16S rRNA gene in the faecal samples and allocated these data into the various taxonomic groups, as described previously by Wijeyesekera et al. ${ }^{10}$. Briefly, total nucleic acid was extracted from faecal samples using the PowerFecal DNA Isolation Kit (MPBio). The V1V2 region of the bacterial 16S rRNA gene was amplified by PCR and sequenced using a MiSeq V3 600 cycle flow cell (Illumina) at the Wellcome Sanger Institute, UK. 16S rRNA gene sequences were quality filtered and analysed using Mothur software ${ }^{56}$.

\section{Bile acid analysis}

Bile acids were extracted from faeces and assayed using liquid chromatography-mass spectrometry (LCMS), following the methods described by Sarafian et al. ${ }^{47}$. Briefly, samples were centrifuged, and supernatant was mixed 1:3 with ice-cold methanol. Reverse-phase chromatography was performed using two mobile phases and a short alkyl (C8) stationary phase adapted from Shockcor et al. ${ }^{57}$. Mobile phase A consisted of acetonitrile and ultra-pure water (1:10), with a final concentration of $1 \mathrm{mM}$ ammonium acetate and adjusted to pH 4.18 using acetic acid, and mobile phase B consisted of acetonitrile and 2propanol (1:1). Bile acid identification was based on the retention times of individual standards.

\section{Statistical analysis}

Data analysis was performed using $\mathrm{R}$ v4.0.2 ${ }^{58}$. Non-parametric variance was calculated using the Kruskal-Wallis rank sum test ${ }^{59}$. Conover-Iman rank sum tests were used to perform multiple comparisons when $\mathrm{H}_{0}$ is rejected by a Kruskal-Wallis test ${ }^{60}$. Inter-group significance displayed in graphs was calculated using the Conover-Iman test. Conover-Iman tests were performed using the conover.test package for $\mathrm{R}^{61}$. Multiple comparisons were corrected using the method proposed by Benjamini and Hochberg, as implemented in conover.test ${ }^{61,62}$. Graphs were created using ggplot2 ${ }^{63}$. Data frame melting was performed with reshape ${ }^{64}$. Shannon diversity indices were calculated using the diversity function from vegan 2.5-6 ${ }^{65}$. Beta-diversity was calculated using the vegdist function of vegan and plotted with ggplot2. Spearman's $\rho$ was calculated using cor.test in $\mathrm{R}^{58}$. Correlation interpretations are based on guidelines published by the British Medical Journal ${ }^{66}$. 


\section{Abbreviations}

12-Dehydrocholic acid (12-DHCA)

23-nor,5ß-Cholanic Acid 3a-,12a-diol (23 nor-5b-CIA,3a,12a-diol)

3-Dehydrocholica acid (3-DHCA)

3-Ketocholanic acid (3-KCIA)

3,12-Diketocholanic acid (3,12-diKCIA)

3a-Hydroxy,12-Ketolithocholic acid (3a-H,12-KLCA)

3a-Hydroxy,12-Ketolithocholic acid (3a-H,12-KLCA)

3a-Hydroxy,6-,7-Diketo-Cholanic acid (3a-H,6,7diKCIA)

5ß-Cholanic acid 3ß-,12a-diol (5b-ClA,3b12A-diol

Chenodeoxycholic acid (CDCA)

Cholic acid (CA)

Deoxycholic_acid (DCA)

Glycochenodeoxycholic acid (GCDA)

Glycoursodeoxycholic acid (GUDCA)

inter-quartile range (IQR)

isolithocholic acid (ILCA)

Liquid Chromatography-Mass Spectrometery (LC-MS)

Lithocholic acid (LCA)

paediatric intensive care units (PICUs)

Recovery Associated Bacteria (RAB)

relative concentrations $(\mathrm{RC})$

Short Chain Fatty Acid (SCFA)

Taurocholic acid (TCA) 
Taurohyocholic acid (THCA)

Tukey's Honest Significant Differences (HSD)

Ursodeoxycholic acid (UDCA)

\section{Declarations}

Ethics approval and consent to participate

Approval for the study was granted by the East Midlands-Nottingham 2 Research Ethics Committee for recruitment from the PICUs at Cambridge University Hospitals NHS Foundation Trust, Great Ormond Street Hospital NHS Foundation Trust, and Imperial College Healthcare NHS Foundation Trust. The City Road and Hampstead Research Ethics committee approved recruitment of healthy children in the Cambridge vicinity. REC reference: 13/LO/0974.

Consent for Publication

Parents of all study subjects provided consent for publication of de-identified data.

\section{Data Availability}

The datasets analysed during the current study are available in the European Nucleotide Archive, https://www.ebi.ac.uk/ena/browser/view/prjeb13830. Data used for analysis can be found in the supplementary files Kean_2021_data_table.csv and Kean_2021_family_taxonomy.csv.

\section{Funding}

Aspects of the work were funded by an Imperial College Biomedical Research Centre award (to Drs. Holmes and Pathan), the Evelyn Trust (to Drs. Parkhill and Pathan), a Wellcome Trust Core Informatics Award (to Dr. Parkhill), Great Ormond Street Hospital Children's Charity (to Drs. Peters

and Ramnarayan), a Wellcome Trust Fellowship (Dr. Baker 215515/Z/19/Z), a Levi-Montalcini award from the European Society of Intensive Care Medicine (to Dr. Pathan) and an Action For Medical Research UK award (Dr. Pathan GN2751). The research was supported by the National Institute for Health Research Biomedical Research Centres based at Cambridge University Hospitals National Health Service (NHS) Foundation Trust, Great Ormond Street Hospital NHS Foundation Trust, Imperial College Healthcare NHS Trust, and Imperial College London. NIHR Cambridge Biomedical Research Centre (BRC-1215-20014)

\section{Conflicts of Interest}

The work in this study is supported by funding to NP from Action Medical Research, the Evelyn Trust and the European Society of Intensive Care Medicine. JP and SB received funding from The Wellcome Trust, 
and JP received funding from Next Gen Diagnostics Llc. The remaining authors have disclosed that they do not have any potential conflicts of interest.

\section{Acknowledgments}

We acknowledge the support of the Imperial College Clinical Phenotyping Centre, a core facility of the National Institute for Health Research Imperial Biomedical Research Centre's Institute of Translational Medicine and Therapeutics. We would like to thank the children and families participating in the study, along with the clinicians treating them. We would like to thank Miss Esther Daubney RNC for her assistance with the archival data. We also thank the core informatics, sequencing, and pathogen informatics teams at the Wellcome Trust Sanger Institute. This research was supported by the NIHR Cambridge Biomedical Research Centre (BRC-1215-20014). The views expressed are those of the authors and not necessarily those of the NIHR or the Department of Health and Social Care

\section{Author contributions}

IRLK conceived the study design, analysed the data and wrote the article. AW developed and supervised the metabolic profiling strategy and substantively revised the article. JW developed and supervised the microbial profiling strategy and substantively revised the article. MDG and JAC substantively revised the article. ST undertook sample processing and data analysis. DKW and JR undertook sample processing, data collection, and analysis. SA, RK, and RO identified and recruited patients. PR contributed to study design and patient data analysis. MJP, and NK contributed to protocol development. JP developed the microbial profiling protocol, supervised all aspects of the microbial data analysis, and edited the article. $\mathrm{EH}$ developed the metabolic profiling protocol and co-wrote the article. SB co-wrote and substantively revised the article. NP conceived and supervised the study and co-wrote the article. All authors read and approved the final manuscript.

\section{Author Affiliations}

Department of Paediatrics, University of Cambridge, Cambridge, United Kingdom.

IRLK, ST, JAC, DKW, JR, SA, RO, NP

The Peter Doherty Institute for Infection and Immunity, Melbourne Health, Melbourne, Australia JW

Wellcome Sanger Institute, Cambridge, Cambridgeshire, United Kingdom.

JW, MdG, JP

Department of Human Microbiome Studies, University of Reading, Berkshire, United Kingdom. AW, MdG 
Department of Veterinary Medicine, University of Cambridge, Cambridge, United Kingdom.

MdG, JP

Department of Haematology, University of Cambridge, Cambridge, United Kingdom.

ST

Cambridge University Hospitals NHS Foundation Trust, Addenbrooke's Hospital, Cambridge Biomedical Campus, Hills Road, Cambridge, United Kingdom

SA, RK, RO, NP

Paediatric Intensive Care Unit, Great Ormond Street Hospital, London, United Kingdom.

PR, MJP, NK

St Mary's Hospital, London, United Kingdom.

PR

UCL Institute of Child Health, Respiratory, Critical Care and Anaesthesia

Section, London, United Kingdom

MJP, NK

Imperial College London, London, United Kingdom.

$\mathrm{EH}$

University of Cambridge School of Clinical Medicine, Cambridge Biomedical Campus, Cambridge

SB

Department of Medicine, University of Cambridge School of Clinical Medicine, Cambridge Biomedical Campus, Cambridge

SB

\section{References}

1. Shao, Y. et al. Stunted microbiota and opportunistic pathogen colonization in caesarean-section birth. Nature 574, 117-121 (2019).

2. Milani, C. et al. The First Microbial Colonizers of the Human Gut: Composition, Activities, and Health Implications of the Infant Gut Microbiota. Microbiol. Mol. Biol. Rev. 81, (2017). 
3. Langdon, A., Crook, N. \& Dantas, G. The effects of antibiotics on the microbiome throughout development and alternative approaches for therapeutic modulation. Genome Medicine vol. 8 (2016).

4. Arrieta, M. C., Stiemsma, L. T., Amenyogbe, N., Brown, E. \& Finlay, B. The intestinal microbiome in early life: Health and disease. Frontiers in Immunology vol. 5 (2014).

5. Stewart, C. J. et al. Temporal development of the gut microbiome in early childhood from the TEDDY study. Nature 562, 583-588 (2018).

6. Ruohtula, T. et al. Maturation of Gut Microbiota and Circulating Regulatory T Cells and Development of IgE Sensitization in Early Life. Front. Immunol. 10, 2494 (2019).

7. Heida, F. H. et al. A Necrotizing Enterocolitis-Associated Gut Microbiota Is Present in the Meconium: Results of a Prospective Study. Clin. Infect. Dis. 62, 863-870 (2016).

8. Rinninella, E. et al. What is the healthy gut microbiota composition? A changing ecosystem across age, environment, diet, and diseases. Microorganisms 7, 14 (2019).

9. Dickson, R. P. The microbiome and critical illness. The Lancet Respiratory Medicine vol. 4 59-72 (2016).

10. Wijeyesekera, A. et al. Multi-Compartment Profiling of Bacterial and Host Metabolites Identifies Intestinal Dysbiosis and Its Functional Consequences in the Critically III Child. Crit. Care Med. 47, e727e734 (2019).

11. Rogers, M. B. et al. Disruption of the microbiota across multiple body sites in critically ill children. Microbiome 4, 66 (2016).

12. Chng, K. R. et al. Metagenome-wide association analysis identifies microbial determinants of postantibiotic ecological recovery in the gut. Nat. Ecol. Evol. 4, (2020).

13. Moron, R. et al. The importance of the microbiome in critically ill patients: Role of nutrition. Nutrients vol. 11 (2019).

14. Moreno-Indias, I. et al. Intermittent hypoxia alters gut microbiota diversity in a mouse model of sleep apnoea. Eur. Respir. J. 45, 1055-1065 (2015).

15. Looft, T. et al. In-feed antibiotic effects on the swine intestinal microbiome. doi:10.1073/pnas.1120238109.

16. Schubert, K., Olde Damink, S. W. M., von Bergen, M. \& Schaap, F. G. Interactions between bile salts, gut microbiota, and hepatic innate immunity. Immunological Reviews vol. 279 23-35 (2017).

17. Urdaneta, V. \& Casadesús, J. Interactions between bacteria and bile salts in the gastrointestinal and hepatobiliary tracts. Frontiers in Medicine vol. 4163 (2017). 
18. Heinken, A. et al. Systematic assessment of secondary bile acid metabolism in gut microbes reveals distinct metabolic capabilities in inflammatory bowel disease. Microbiome 7, 75 (2019).

19. Ridlon, J. M., Kang, D. J. \& Hylemon, P. B. Bile salt biotransformations by human intestinal bacteria. Journal of Lipid Research vol. 47 241-259 (2006).

20. Kang, J. D. et al. Bile Acid 7a-Dehydroxylating Gut Bacteria Secrete Antibiotics that Inhibit Clostridium difficile: Role of Secondary Bile Acids. Cell Chem. Biol. 26, 27-34.e4 (2019).

21. Solbach, P. et al. BaiCD gene cluster abundance is negatively correlated with Clostridium difficile infection. PLOS ONE 13, e0196977 (2018).

22. Staley, C., Weingarden, A. R., Khoruts, A. \& Sadowsky, M. J. Interaction of gut microbiota with bile acid metabolism and its influence on disease states. Applied Microbiology and Biotechnology vol. 101 47-64 (2017).

23. Ridlon, J. M., Kang, D. J., Hylemon, P. B. \& Bajaj, J. S. Bile acids and the gut microbiome. Current Opinion in Gastroenterology vol. 30 332-338 (2014).

24. Ridlon, J. M., Wolf, P. G. \& Gaskins, H. R. Taurocholic acid metabolism by gut microbes and colon cancer. Gut Microbes vol. 7 201-215 (2016).

25. Ma, H., Patti, M. E. \& Endocrinologist, A. Bile acids, obesity, and the metabolic syndrome. Best Practice and Research: Clinical Gastroenterology vol. 28 573-583 (2014).

26. Festi, D. et al. Gut microbiota and metabolic syndrome. World Journal of Gastroenterology vol. 20 16079-16094 (2014).

27. Reddy, B. S., Narasawa, T., Weisburger, J. H. \& Wynder, E. L. Promoting Effect of Sodium Deoxycholate on Colon Adenocarcinomas in Germfree Rats2. JNCI J. Natl. Cancer Inst. 56, 441-442 (1976).

28. Reddy, B. S., Watanabe, K., Weisburger, J. H. \& Wynder, E. L. Promoting Effect of Bile Acids in Colon Carcinogenesis in Germ-free and Conventional F344 Rats. Cancer Res. 37, (1977).

29. Attene-Ramos, M. S., Wagner, E. D., Gaskins, H. R. \& Plewa, M. J. Hydrogen sulfide induces direct radical-associated DNA damage. Mol. Cancer Res. 5, 455-459 (2007).

30. Devkota, S. et al. Dietary-fat-induced taurocholic acid promotes pathobiont expansion and colitis in II10-/- mice. Nature vol. 487 104-108 (2012).

31. Shetty, S. A. et al. Reclassification of Eubacterium hallii as Anaerobutyricum hallii gen. nov., comb. nov., and description of Anaerobutyricum soehngenii sp. nov., a butyrate and propionate-producing bacterium from infant faeces. Int. J. Syst. Evol. Microbiol. 68, 3741-3746 (2018). 
32. Togo, A. H. et al. Description of Mediterraneibacter massiliensis, gen. nov., sp. nov., a new genus isolated from the gut microbiota of an obese patient and reclassification of Ruminococcus faecis, Ruminococcus lactaris, Ruminococcus torques, Ruminococcus gnavus and Clostridium glycyrrhizinilyticum as Mediterraneibacter faecis comb. nov., Mediterraneibacter lactaris comb. nov., Mediterraneibacter torques comb. nov., Mediterraneibacter gnavus comb. nov. and Mediterraneibacter glycyrrhizinilyticus comb. nov. Antonie Van Leeuwenhoek 111, 2107-2128 (2018).

33. Hirano, S., Nakama, R., Tamaki, M., Masuda, N. \& Oda, H. Isolation and characterization of thirteen intestinal microorganisms capable of 7a-dehydroxylating bile acids. Appl. Environ. Microbiol. 41, 737745 (1981).

34. Berr, F., Kullak-Ublick, G. A., Paumgartner, G., Monzing, W. \& Hylemon, P. B. 7a-Dehydroxylating bacteria enhance deoxycholic acid input and cholesterol saturation of bile in patients with gallstones. Gastroenterology 111, 1611-1620 (1996).

35. Chiang, J. Y. L. Bile acids: Regulation of synthesis. J. Lipid Res. 50, 1955-1966 (2009).

36. Gibbons, S. M. Keystone taxa indispensable for microbiome recovery. Nat. Microbiol. 5, 10671068 (2020).

37. Paine, R. t. A Conversation on Refining the Concept of Keystone Species. Conserv. Biol. 9, 962-964 (1995).

38. Begley, M., Hill, C. \& Gahan, C. G. M. Bile salt hydrolase activity in probiotics. Applied and Environmental Microbiology vol. 72 1729-1738 (2006).

39. Vital, M., Rud, T., Rath, S., Pieper, D. H. \& Schlüter, D. Diversity of Bacteria Exhibiting Bile Acidinducible 7a-dehydroxylation Genes in the Human Gut. Comput. Struct. Biotechnol. J. 17, 1016-1019 (2019).

40. Marion, S. et al. In vitro and in vivo characterization of Clostridium scindens bile acid transformations. Gut Microbes 10, 481-503 (2018).

41. Duncan, S. H., Louis, P. \& Flint, H. J. Lactate-Utilizing Bacteria, Isolated from Human Feces, That Produce Butyrate as a Major Fermentation Product. Appl. Environ. Microbiol. 70, 5810-5817 (2004).

42. PICANet_2019_Annual_Report_Tables_and_Figures_v1.0.pdf.

43. Neu, J. \& Rushing, J. Cesarean Versus Vaginal Delivery: Long-term Infant Outcomes and the Hygiene Hypothesis. Clinics in Perinatology vol. 38 321-331 (2011).

44. Biasucci, G., Benenati, B., Morelli, L., Bessi, E. \& Boehm, G. Cesarean delivery may affect the early biodiversity of intestinal bacteria. in Journal of Nutrition (2008). doi:10.1093/jn/138.9.1796s. 
45. Dominguez-Bello, M. G. et al. Partial restoration of the microbiota of cesarean-born infants via vaginal microbial transfer. Nat. Med. 22, 250-253 (2016).

46. Yassour, M. et al. Natural history of the infant gut microbiome and impact of antibiotic treatment on bacterial strain diversity and stability. Sci. Transl. Med. 8, 343ra81-343ra81 (2016).

47. Sarafian, M. H. et al. Bile Acid Profiling and Quantification in Biofluids Using Ultra-Performance Liquid Chromatography Tandem Mass Spectrometry. Anal. Chem. 87, 9662-9670 (2015).

48. Kakiyama, G. et al. A simple and accurate HPLC method for fecal bile acid profile in healthy and cirrhotic subjects: Validation by GC-MS and LC-MS. J. Lipid Res. 55, 978-990 (2014).

49. Strathmann, F. G. \& Hoofnagle, A. N. Current and Future Applications of Mass Spectrometry to the Clinical Laboratory. Am. J. Clin. Pathol. 136, 609-616 (2011).

50. Seger, C. \& Salzmann, L. After another decade: LC-MS/MS became routine in clinical diagnostics. Clinical Biochemistry vol. 82 2-11 (2020).

51. Bokulich, N. A. et al. Antibiotics, birth mode, and diet shape microbiome maturation during early life. Sci. Transl. Med. 8, 343ra82 (2016).

52. Wang, C. et al. Effects of oral administration of Bifidobacterium breve on fecal lactic acid and short-chain fatty acids in low birth weight infants. J. Pediatr. Gastroenterol. Nutr. (2007) doi:10.1097/01.mpg.0000252184.89922.5f.

53. Suez, J. et al. Post-Antibiotic Gut Mucosal Microbiome Reconstitution Is Impaired by Probiotics and Improved by Autologous FMT. Cell 174, 1406-1423.e16 (2018).

54. Mallick, H. et al. Predictive metabolomic profiling of microbial communities using amplicon or metagenomic sequences. Nat. Commun. 10,1-11 (2019).

55. Larsen, P. E. \& Dai, Y. Metabolome of human gut microbiome is predictive of host dysbiosis. GigaScience (2015) doi:10.1186/s13742-015-0084-3.

56. Schloss, P. D. et al. Introducing mothur: Open-source, platform-independent, community-supported software for describing and comparing microbial communities. Appl. Environ. Microbiol. 75, 7537-7541 (2009).

57. Shockcor, J., Crowe, H., Yu, K. \& Shion, H. Analysis of Intact Lipids from Biologics Matrices by UPLC / Ion Mobility TOF-MS. Waters Corp. Milford MA USA 2-4 (2011).

58. R Core Team. R software: Version 4.0.2. R Found. Stat. Comput. (2020).

59. Kruskal, W. H. \& Wallis, W. A. Use of Ranks in One-Criterion Variance Analysis. J. Am. Stat. Assoc. 47, 583-621 (1952). 
60. Conover, W. J. \& Iman, R. L. Rank Transformations as a Bridge Between Parametric and Nonparametric Statistics. Am. Stat. 35, 124 (1981).

61. Dinno, A. conover.test: Conover-Iman Test of Multiple Comparisons Using Rank Sums. (2017).

62. Benjamini, Y. \& Hochberg, Y. Controlling the False Discovery Rate: A Practical and Powerful Approach to Multiple Testing. J. R. Stat. Soc. Ser. B Methodol. 57, 289-300 (1995).

63. Wickham, H. ggplot2 Elegant Graphics for Data Analysis (Use R!). Springer(2016). doi:10.1007/978-0-387-98141-3.

64. Wickham, H. R: Package 'reshape2'. CRAN(2017).

65. Oksanen, J. et al. vegan: Community Ecology Package. R package version 2.5-2. Cran R(2019).

66. 11. Correlation and regression / The BMJ. The BMJ / The BMJ: leading general medical journal. Research. Education. Comment https://www.bmj.com/about-bmj/resourcesreaders/publications/statistics-square-one/11-correlation-and-regression (2020).

\section{Figures}

\section{Figure 1}

Primary and secondary bile acids a) primary bile acids (Cholic acid (CA), Chenodeoxycholic acid (CDCA), Glycochenodeoxycholic acid (GCDCA), and Taurocholic acid (TCA)) in faecal water measured by LC-MS as a ratio of total measured bile acid. b) secondary bile acids (Deoxycholic Acid (DCA), Lithocholic acid (LCA), Isolithocholic acid (ILCA), Ursodeoxycholic acid (UDCA), 3囚-Hydroxy,12-Ketolithocholic acid (3aH,12-KLCA), Taurohyocholic acid (THCA), and Glycoursodeoxycholic acid (GUDCA)) in faecal water measured by LC-MS as a ratio of total measured bile acid. A significant reduction to the relative concentration of total secondary bile acids versus the total measured bile acid pool was observed for patients sampled at day 1-3 and day 4-7 compared to the control. The median proportion of these secondary bile acids at days 8-10 was comparable to that of the aged-matched controls, but we observed a significant increase of patient bile acids at days 8-10 above the median for patients at day 1-3. c) the ratio of CA to CDCA, two species of primary bile acids in the human gut. The control samples had a median CA:CDCA ratio of 0.9; the median CA:CDCA ratios on days 1-3, 4-7, an 8-10 in the faecal samples of the critically patients were 4.4,4.4, and 5.9, respectively. d) Ratio of primary bile acids to secondary bile acids. e) ratio of CA to DCA in faecal water.The ratio of CA to DCA was elevated in across all patient timepoints compared to the control. Patient samples collected on days 8-10 had lower ratios of CA:DCA than patients at day 1-3. f) ratio of CDCA to LCA and ILCA. CDCA:LCA ratios were increased compared to 
controls in patient samples from day 1-3 and day 4-7, returning to control levels at days 8-10. ConoverIman significance ${ }^{* \star} p \leq 0.001,{ }^{* *} p \leq 0.01, * p \leq 0.05, p>0.05=$ not shown.
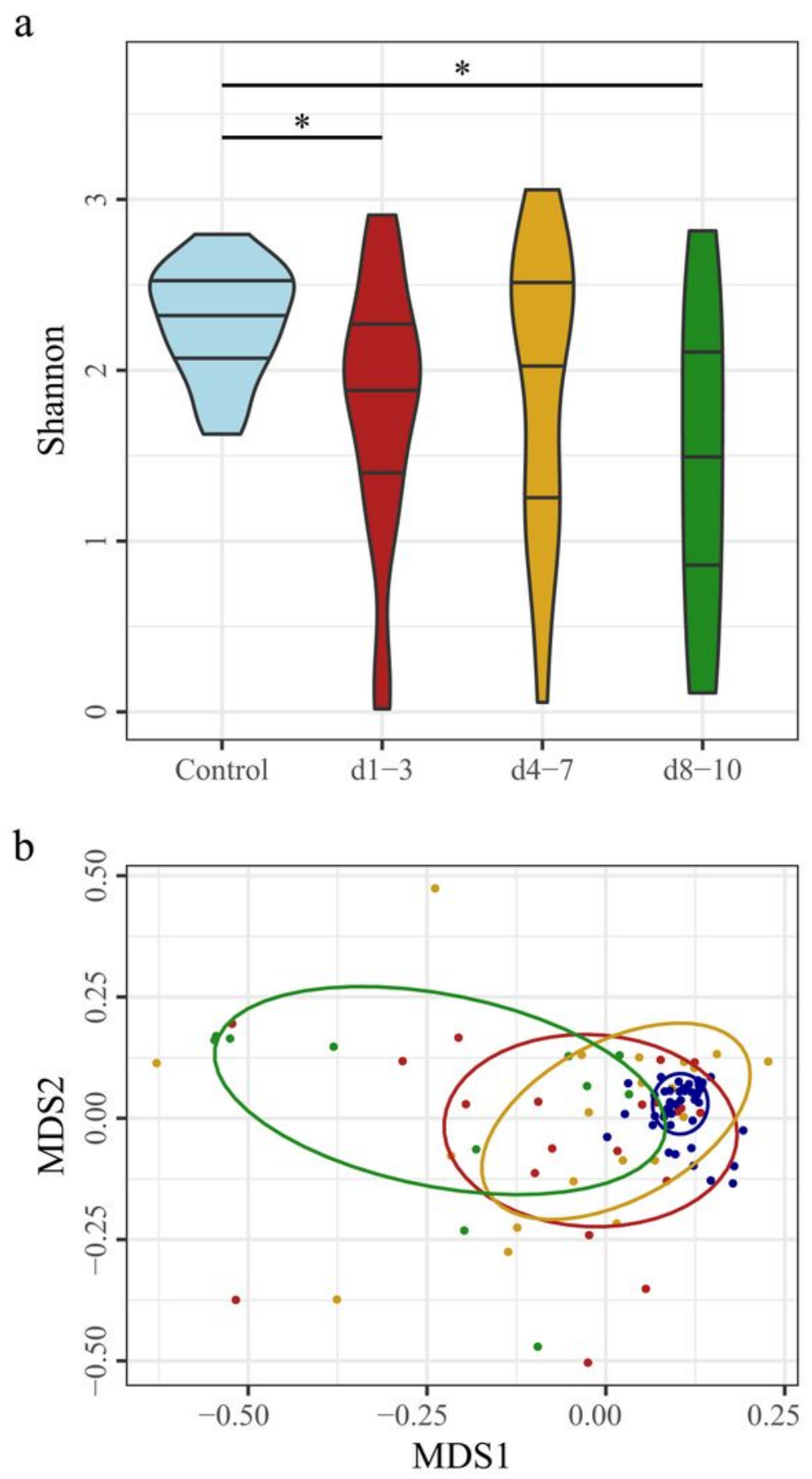

$\rightarrow$ Control $\rightarrow \mathrm{d} 1-3 \rightarrow \mathrm{d} 4-7 \rightarrow \mathrm{d} 8-10$

Figure 2

Bacterial diversity, and abundance of beneficial bacteria. a) Shannon diversity of sampling groups, bars indicate 1 st 2 nd and 3 rd quartiles. Due to the broad range of alpha-diversity at day 1-3, day 4-7 and days 8-10, only the median alpha diversity of samples collected at days 8-10 was significantly lower than that 
of the control group after correcting for multiple comparisons. b) NMDS clustering of beta diversity of bacterial populations compared between sampling groups. PERMANOVA $p$-value $=0.001$. Ellipses indicate $60 \%$ similarity. Conover-Iman significance ${ }^{\star \star *} p \leq 0.001$, $* * p \leq 0.01, * p \leq 0.05, p>0.05=$ not shown.

\section{Figure 3}

Relative proportion of bacteria identified by 16S rRNA gene sequencing. Bacterial 16S rRNA gene counts are represented as proportional abundance at Family level for each sample. The majority of analysed $16 \mathrm{~S}$ rRNA gene counts are within $12 \mathrm{k}$ to $16 \mathrm{k}$ reads. The ten most abundant families in the control microbiomes were the Bacteroidaceae, Ruminococcaceae, Prevotellaceae, Lachnospiraceae, Tannerellaceae, Veillonellacea, Peptostreptococcaceae, Coriobacteriaceae, Rikenellaceae and Acidaminococcaceae. These ten families represent a median of $82.8 \%$ (IQR 12.7\%) of the control faecal microbiota and a median of $45.3 \%$ (IQR $57 \%$ ) of the patient faecal microbiota. The ten most abundant bacterial families present in the patient faecal microbiome, which were not highly represented in the control samples were the Enterococcaceae, Enterobacteriacea, Bifidobacteriaceae, Streptococcaceae, Staphylococcaceae, Corynebacteriaceae, Clostridiaceae, Lactobacillaceae, Christensenellaceae and the Sutterellaceae. These families represented a median of 7.9\% (IQR $8.8 \%$ ) of the control microbiome, and a median of $31.1 \%$ (IQR $64.8 \%$ ) of the patient samples.

\section{Figure 4}

Relative abundance of recovery associated bacteria (RAB) and Lachnospiraceae. a) Proportion of RAB present in microbiomes. Patient samples from day 1-3, day 4-7 and days 8-10 were significantly reduced from the median level of the control group. RAB at days 8-10 were also significantly reduced from the median RAB level at day 4-7. b) Proportion of Lachnospiraceae present in microbiomes. No significant difference was observed between groups after controlling for multiple comparisons. Conover-Iman significance *** $p \leq 0.001, * \star p \leq 0.01, * p \leq 0.05, p>0.05=$ not shown.

\section{Supplementary Files}

This is a list of supplementary files associated with this preprint. Click to download.

- Kean2021FigureS1.eps

- Kean2021FigureS2.eps

- Kean2021FigureS3.eps

- Kean2021FigureS4.eps

- Kean2021 supplementarytables.docx 
- Kean2021Rscripts.rmd

- Kean2021datatable.csv

Page 23/23 